\title{
Chemical Identification at the Solid-Liquid Interface
}

Hagen Söngen, ${ }^{\dagger \dagger}$ Christoph Marutschke, ${ }^{\dagger}$ Peter Spijker, ${ }^{\S}$ Eric Holmgren, ${ }^{\dagger}$ Ilka Hermes, ${ }^{\dagger}$ Ralf Bechstein, ${ }^{\dagger}$ Stefanie Klassen, ${ }^{\dagger}$ John Tracey, ${ }^{\S}$ Adam S. Foster, ${ }^{\S, \perp}$ and Angelika Kühnle*,†

${ }^{\dagger}$ Institute of Physical Chemistry, Johannes Gutenberg University Mainz, Duesbergweg 10-14, 55099 Mainz, Germany

${ }^{\ddagger}$ Graduate School Materials Science in Mainz, Staudingerweg 9, 55128 Mainz, Germany

${ }^{\S}$ COMP Centre of Excellence, Department of Applied Physics, Aalto University, Helsinki FI-00076, Finland

"University of Rochester, Rochester, New York 14627, United States

${ }^{\perp}$ Division of Electrical Engineering and Computer Science, Kanazawa University, Kanazawa 920-1192, Japan

\section{Supporting Information}

ABSTRACT: Solid-liquid interfaces are decisive for a wide range of natural and technological processes, including fields as diverse as geochemistry and environmental science as well as catalysis and corrosion protection. Dynamic atomic force microscopy nowadays provides unparalleled structural insights into solid-liquid interfaces, including the solvation structure above the surface. In contrast, chemical identification of individual interfacial atoms still remains a considerable challenge. So far, an identification of chemically alike atoms in a surface alloy has only been demonstrated under well-controlled ultrahigh vacuum conditions. In liquids, the recent advent of three-dimensional force mapping has opened

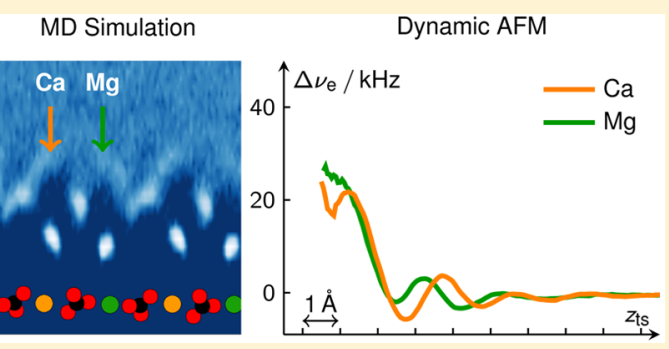
the potential to discriminate between anionic and cationic surface species. However, a full chemical identification will also include the far more challenging situation of alike interfacial atoms (i.e., with the same net charge). Here we demonstrate the chemical identification capabilities of dynamic atomic force microscopy at solid-liquid interfaces by identifying Ca and $\mathrm{Mg}$ cations at the dolomite-water interface. Analyzing site-specific vertical positions of hydration layers and comparing them with molecular dynamics simulations unambiguously unravels the minute but decisive difference in ion hydration and provides a clear means for telling calcium and magnesium ions apart. Our work, thus, demonstrates the chemical identification capabilities of dynamic AFM at the solid-liquid interface.

\section{INTRODUCTION}

Dynamic atomic force microscopy ${ }^{1,2}$ (AFM) has developed into a most versatile tool that is capable of imaging surfaces with atomic resolution not only in a well-controlled ultrahigh vacuum (UHV) environment ${ }^{3}$ but also at the solid-liquid interface. ${ }^{4}$ When analyzing distance-dependent data, dynamic AFM can even provide chemical information on individual atoms at the surface, as has first been demonstrated in UHV: force-distance curves have been collected on $\operatorname{Si}(111)-(7 \times 7)$, revealing site-specific differences and, thus, demonstrating the potential to differentiate inequivalent adatoms by a careful analysis of force-distance curves. ${ }^{5}$ Later, ionic crystal sublattices have been identified on several crystals, including, for example, $\mathrm{CaF}_{2}(111),{ }^{6} \mathrm{NiO}(001),{ }^{7,8} \mathrm{NaCl}(001),{ }^{9}$ and calcite (10.4).$^{10}$ A particularly challenging system has been studied by Sugimoto et al. who have been the first to demonstrate the identification of individual surface atoms in an alloy of $\mathrm{Pb}, \mathrm{Sn}$, and $\mathrm{Si}$, even though these atoms occupy identical surface positions and are not oppositely charged. ${ }^{11}$ All the above studies have been carried out in UHV.

At the solid-liquid interface, however, the situation is usually significantly more complex due to the presence of the solvent molecules that can form a solvation structure not only at the surface but also at the probe tip. ${ }^{12}$ Nevertheless, due to the omnipresence of water films on surfaces, especially the solidwater interface has attracted considerable attention in the last decades $^{13-16}$ with the first demonstration of atomic-scale imaging with dynamic AFM on a mica (001) surface in 2005 by Fukuma et al. ${ }^{4}$ Using conventional imaging on calcite (10.4), ionic sublattices have been assigned based on the different lateral structure. ${ }^{17}$ Recently, the development of three-dimensional (3D) force mapping in liquids ${ }^{18}$ has considerably pushed this field of research. Three-dimensional force maps have been collected on, for example, calcite $(10.4),{ }^{19,20}$ mica, ${ }^{18,21} \alpha$ $\mathrm{Al}_{2} \mathrm{O}_{3}{ }^{22}$ graphite, ${ }^{23}$ alkanethiol films, ${ }^{24,25}$ and organic crystals. ${ }^{26}$ Analyzing site-specific differences in the force-distance curves has allowed for the discrimination between anionic and cationic surface species. ${ }^{20}$ So far, however, the identification of chemically alike interfacial species possessing the same net charge has not been demonstrated. Such a differentiation is, however, an essential prerequisite for chemical identification, one of the major challenges of surface science. For such a

Received: October 19, 2016

Revised: December 9, 2016

Published: December 13, 2016 


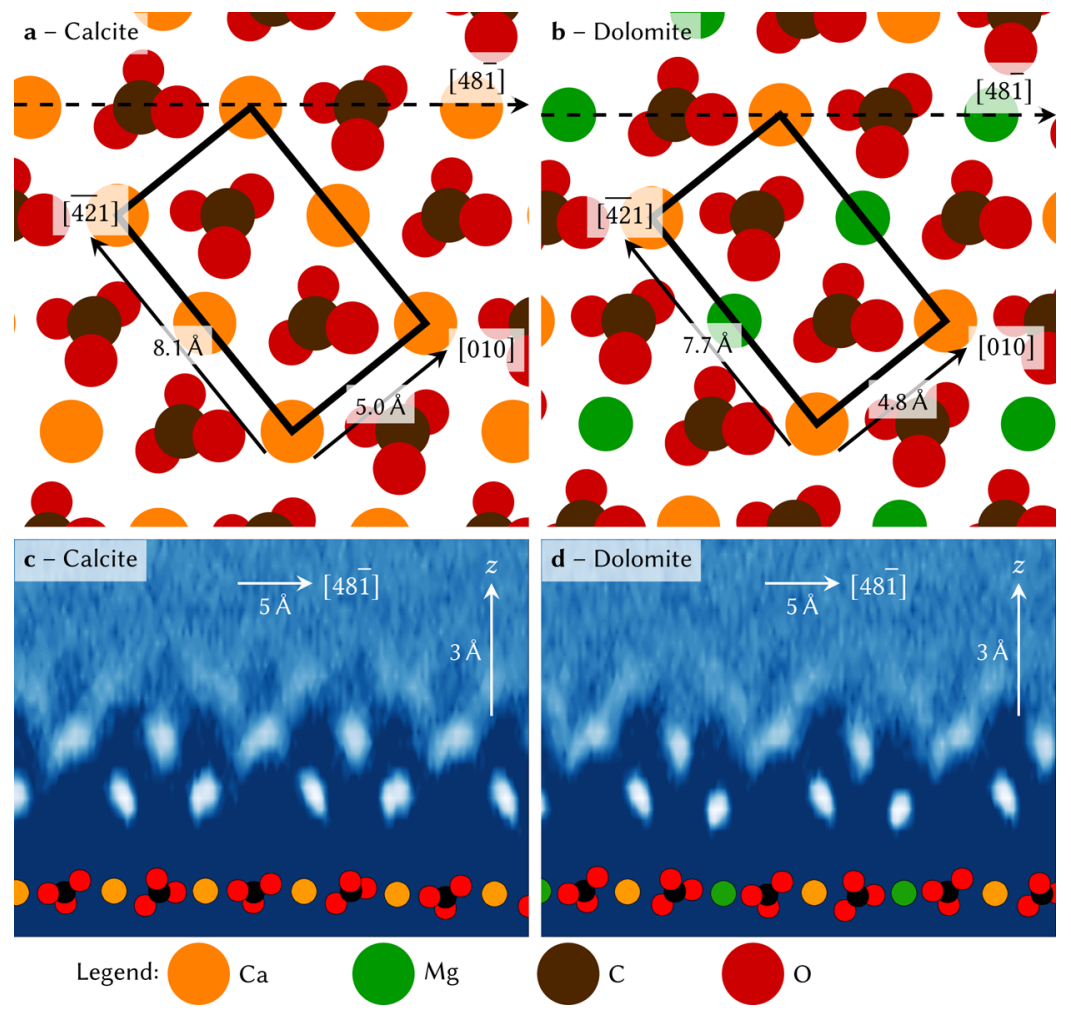

Figure 1. Interfacial water on calcite and dolomite. Schematic model of the (10.4) surface of calcite (a) and dolomite (b). The unit cell dimensions are $8.1 \AA \times 5.0 \AA$ and $7.7 \AA \times 4.8 \AA$, respectively. The two carbonate groups in the surface unit cell are tilted with respect to the surface. As the protruding oxygen atom of the carbonate group points in alternating directions, the carbonate groups are not equivalent. The lower panel shows the

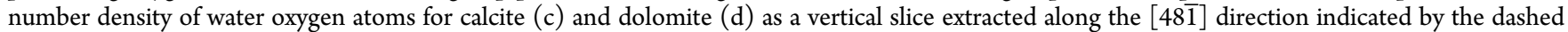
line shown in (a) and (b). The color scale ranges from dark blue (low density) to white (high density).

demonstration, we investigate an ideally suited model system, namely dolomite(10.4), possessing two chemically alike cation species that can be benchmarked against the well-studied calcite (10.4) surface. Here, by comparing high-resolution threedimensional (3D) AFM measurements with molecular dynamics (MD) simulations, we show that the different hydration of $\mathrm{Mg}$ ions as compared to $\mathrm{Ca}$ ions leads to a shift of interfacial water above $\mathrm{Mg}$ ions that allows for their discrimination from $\mathrm{Ca}$ ions on the surface of dolomite.

\section{METHODS}

Experimental Section. Experiments were performed with a custom 3D-AFM ${ }^{27-29}$ operated in the frequency-modulation mode. ${ }^{1}$ Calcite $\left[\mathrm{CaCO}_{3}\right]$ crystals (Korth Kristalle, Germany) and dolomite $\left[\mathrm{CaMg}\left(\mathrm{CO}_{3}\right)_{2}\right]$ crystals (SurfaceNet, Germany) were cleaved prior to the measurement. We used Si cantilevers exhibiting an eigen frequency of approximately $150 \mathrm{kHz}$, a quality factor of approximately 8 and a spring constant of approximately $40 \mathrm{~N} \mathrm{~m}^{-1}$ in liquids (types PPPNCHAuD, Tap300G, and Tap300GB-G were used). Oscillation amplitudes in the order of $0.7-0.8 \AA$ have been used. All AFM measurements were performed in pure water (MilliPore). The deflection sensitivity was determined from static deflection versus piezo-displacement curves, and the spring constant by evaluation of the thermal noise. ${ }^{30}$ The crystallographic surface directions were obtained from the direction of birefringence. ${ }^{31}$ Experimental data sets were corrected for sample tilt and vertical drift as described previously. ${ }^{29}$ Moreover, data were corrected for lateral drift by comparison of up and down scans to identify the surface unit cell. Subsequently, the images were adjusted so that the surface unit cell dimensions correspond to the unit cell dimensions determined from the dimension of the bulk unit cell. We obtained the averaged tipsample force gradient ${ }^{2}\left\langle k_{\mathrm{ts}}\right\rangle_{\cap}$ according to

$$
\left\langle k_{\mathrm{ts}}\right\rangle_{\cap}=k\left(1-\frac{\nu_{\mathrm{exc}}^{2}}{\nu_{\mathrm{e}}^{2}}\right)-\frac{F_{0}}{A^{\prime}} \cos \varphi^{\prime}
$$

where $k$ is the spring constant, $\nu_{\mathrm{e}}$ is the eigen frequency of the cantilever (far away from the surface), $\nu_{\text {exc }}$ is the excitation frequency (the measured eigen frequency shift is $\Delta \nu_{\mathrm{e}}=\nu_{\mathrm{exc}}-\nu_{\mathrm{e}}$ ), $F_{0}$ is the excitation force amplitude, $A^{\prime}$ is the oscillation amplitude, and $\varphi^{\prime}$ is the phase shift between cantilever oscillation and excitation. Deconvolution $^{32}$ of $\left\langle k_{\mathrm{ts}}\right\rangle_{\mathrm{n}}$ yielded the even contribution to the tip-sample force. ${ }^{33,34}$ Both quantities are shown in Supporting Figure S3.

Simulations. For all simulations, the large scale molecular dynamics code LAMMPS $^{35}$ was used. The simulations were run in parallel on a typical Linux commodity cluster, and analysis was performed visually using $\mathrm{VMD}^{36}$ or numerically using the Python library MDAnalysis. ${ }^{37}$ In order to model the crystal structure of the (10.4) cleavage plane, we used a crystal that is seven layers thick and where each layer consists of five unit cells along the [421] and eight along the [010] direction. For dolomite, the simulation box dimensions were scaled down (while keeping the internal carbonate bonds at the correct length) with respect to the dimensions for calcite in order to match the surface unit cell size. Each simulation consists of a similar protocol. First, the seven-layer crystal is modeled as if it is a bulk crystal in order to relax our initial scaling, during which no external constraints other than a common barostat and thermostat were applied to the atoms. Typically, these seven-layer crystals measure $4.06 \mathrm{~nm} \times 3.9 \mathrm{~nm} \times 2.13 \mathrm{~nm}$ (calcite) and $3.89 \mathrm{~nm} \times 3.82$ $\mathrm{nm} \times 2.05 \mathrm{~nm}$ (dolomite). Subsequently, the crystal is placed in a larger box and solvated on either side of the (10.4) surface by ample water, such that far away from the surface bulk properties can be reached, adding approximately $16 \mathrm{~nm}$ in the direction perpendicular to the surface. The total number of atoms in each of the simulations is 28234 for calcite and 26254 for dolomite. In the next steps, the lateral dimensions of the simulation box were fixed, along with the carbon 

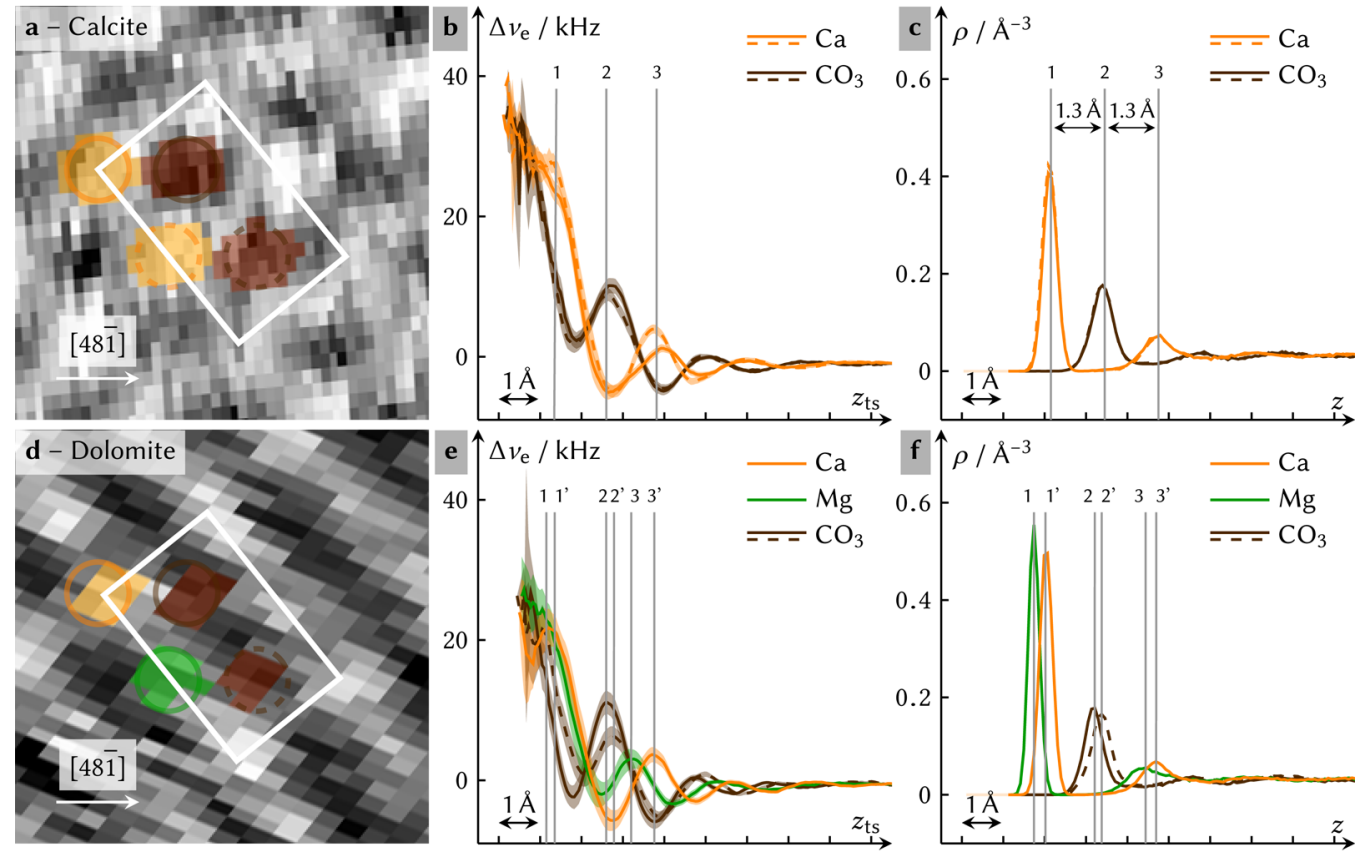

Figure 2. Comparison of experimental and theoretical hydration data for calcite (upper part) and dolomite (lower part). Drift-corrected lateral slices of eigen frequency shift $\left(\Delta \nu_{\mathrm{e}}\right)$ maps are presented in the first column $(\mathrm{a}, \mathrm{d})$. Eigen frequency shift versus tip-sample distance $\left(z_{\mathrm{ts}}\right)$ curves extracted above the different sites indicated in the first column are shown in the second column $(b, e)$. In $(c, f)$, site-specific density curves from the MD simulations are shown. Yellow, green, and brown color indicate $\mathrm{Ca}, \mathrm{Mg}$, and $\mathrm{CO}_{3}$ sites, respectively. Dashed curves in the graphs correspond to the lower site indicated in the lateral slices. The shaded area below and above the curves in $(\mathrm{b}, \mathrm{e})$ indicate a $95 \%$ confidence interval determined from the standard deviation $s$ and the number of samples $n$ according to $\pm 1.96 s / \sqrt{ } n$. The site-specific density profiles shown in $(\mathrm{c}, \mathrm{f})$ result from averaging individual density profiles within a circle of $1.25 \AA$ radius centered on each respective ion. Each (symmetry equivalent) ion within the simulation box was considered.

atoms of the center-most layer in order to ensure the proper crystal dimensions and no thermal drift of the system. First, the entire system is allowed to relax for at least $50 \mathrm{ps}$ (using a $1 \mathrm{fs}$ time step) at ambient conditions $(310 \mathrm{~K}$ and $1013.25 \mathrm{hPa})$. After that, a longer run $(0.5 \mathrm{~ns})$ is performed to allow for the hydration layers to form. Following this, the unit cell dimension along $z$ as well as the total and separate energy components were constant (except for thermal oscillations), confirming that equilibrium has been reached. The next $8 \mathrm{~ns}$ are the simulation production run, where each 2.5 ps a snapshot of the system is saved to disk and used for subsequent analysis.

An accurate force field for calcite simulations was developed by Raiteri et al., and has been used successfully in simulating the growth of calcium carbonate in aqueous solutions. ${ }^{38}$ Here, we used the same force field, except that we replace the intramolecular angle and improper terms for the carbonates by more common harmonic potentials providing equivalent interactions. Magnesium terms were taken from the extended potentials of Tomono et al., which allows for the modeling of dolomite. ${ }^{39}$ This proved to show no significant differences in the calculated density to more recently published potentials. ${ }^{40}$ For water, we used the single point charge flexible model $(\mathrm{SPC} / \mathrm{Fw}) .{ }^{41}$

\section{RESULTS AND DISCUSSION}

Figure 1a,b shows an atomistic model of the (10.4) surfaces of calcite and dolomite, respectively. In both cases, the surface unit cell (black rectangle) contains two cations and two carbonate groups. In the case of calcite, the cations are $\mathrm{Ca}$ and in case of dolomite the surface unit cell contains both a $\mathrm{Ca}$ and a $\mathrm{Mg}$ ion, which leads to alternating $\mathrm{Ca}$ and $\mathrm{Mg}$ ions oriented along the [421] direction. Therefore, the solid-liquid interface of the dolomite (10.4) surface constitutes an ideal test system to assess the feasibility of chemical identification of the two equally charged cations. To obtain the water density in the volume above the two substrates, we performed molecular dynamics (MD) simulations. In Figure $1 c, d$, we show the density of water oxygen atoms extracted along a row of alternating cations and anions (dashed line in Figure 1a,b) for calcite and dolomite, respectively. In the case of calcite, we find that water forms a laterally as well as a vertically ordered structure above the surface. The water molecules in the first layer are located above calcium ions and the ones in the second layer are above carbonate sites. This alternating arrangement of interfacial water above the cations and anions continues in several layers and leads to a characteristic checkerboard-like pattern. Our simulations agree with previous theoretical studies on interfacial water above calcite. ${ }^{20,42,43}$

For dolomite, the simulated water density shown in Figure 1d shows a similar checkerboard-like pattern of water density maxima. Similar to calcite, the first layer is placed above the cations, which are now $\mathrm{Ca}$ and $\mathrm{Mg}$. However, although $\mathrm{Ca}$ and $\mathrm{Mg}$ cations occupy virtually identical positions in the crystal lattice, the oxygen water density maximum above $\mathrm{Mg}$ sites is shifted closer to the surface compared to the Ca site. This finding can be rationalized by the smaller size and, consequently, the larger charge density of $\mathrm{Mg}$ compared to Ca. ${ }^{44}$

Next, we explore whether we can make use of this subtle difference to provide chemical identification of these two interfacial cations on the surface of dolomite. To address this challenging task, we have optimized a commercial AFM setup for performing high-resolution imaging at the solid-liquid interface $^{27,28}$ and added a highly flexible routine for collecting 3D AFM data. ${ }^{29}$ As reference, we first analyze distancedependent data on calcite at four sites (two above the cations, 
two above the anions) within the calcite surface unit cell. A lateral slice of a $3 \mathrm{D}$ eigen frequency shift $\left(\Delta \nu_{\mathrm{e}}\right)$ data set obtained on calcite in pure water is shown in Figure 2a. We ensure that this lateral slice is extracted within a hydration layer, as is explained in detail in the Supporting Information. In Figure $2 \mathrm{~b}$, we show site-specific $\Delta \nu_{\mathrm{e}}\left(z_{\mathrm{ts}}\right)$ curves extracted at the two minima and the two maxima observed within each surface unit cell by averaging over the areas indicated by the colored overlays in Figure 2a. For clarity, we only show this overlay for one surface unit cell, all extraction sites (which were obtained by translations according to the surface unit cell dimensions) are shown in Supporting Figure S2. A clear agreement between the two curves extracted at sites that are shifted by half a unit cell length along the [ $\overline{42} 1]$ direction can be observed (both colored either yellow or brown, respectively). Moreover, all four extracted curves exhibit an oscillatory shape. We interpret each maximum (minimum) in the frequency shift as a maximum (minimum) in the water density, which is in line with the solvent tip approximation ${ }^{45-47}$ (STA). Details on the STA are discussed in the Supporting Information. Note that the eigen frequency shift close to the surface shows an overall increase that it interpreted by increasingly repulsive interactions originating from the presence of a rigid surface solely.

Next, we identify whether the curves were extracted above $\mathrm{Ca}$ or $\mathrm{CO}_{3}$ sites. By comparison with the simulated water density [Figure 1c], the curve with the minimum in the frequency shift at the smallest tip-sample distance (brown curves) can be readily assigned to a $\mathrm{CO}_{3}$ ion, while the other set of curves (yellow curves) is assigned to $\mathrm{Ca}$ ions. The excellent agreement between the curves extracted above $\mathrm{Ca}$ and $\mathrm{CO}_{3}$ sites, respectively, is confirmed by identical water density curves obtained by MD simulations (Figure 2c). The obtained vertical distance between two water density maxima is $1.3 \AA$ [arrows in Figure 2c]. This distance agrees remarkably well with the layerto-layer distances for the presented data in Figure $2 \mathrm{~b}$. As our description is based on the simple STA model deviations between the theoretically obtained and the experimentally determined layer-to-layer distances can be expected.

After having analyzed calcite as a reference, we applied the same analysis protocol to a $3 \mathrm{D}$ data set obtained on the dolomite (10.4) surface. Here, $\Delta \nu_{\mathrm{e}}\left(z_{\mathrm{ts}}\right)$ curves extracted at the sites indicated in Figure $2 \mathrm{~d}$ are presented in Figure 2e. The individual curves exhibit a very similar shape compared to calcite as they also show alternating extrema. Two of the four curves (extracted at sites shifted half a unit-cell along the [ $\overline{42} 1]$ direction) exhibit a clear minimum at the smallest tip-sample distance and are, consequently, again identified as $\mathrm{CO}_{3}$ sites (brown color). In clear contrast to calcite, however, the other two curves, extracted above the cation sites, show a distinctive shift relative to each other. This shift is also reflected in the MD simulations (Figure 2f), as water molecules are located closer to $\mathrm{Mg}$ ions compared to $\mathrm{Ca}$ ions. Therefore, we assign the cationsite curve that is shifted more closely to the surface (green, without prime) to a magnesium site, while the other site (yellow, with primes) is assigned to a $\mathrm{Ca}$ site. This is a demonstration of chemical identification of interfacial ions possessing the same charge. Note that even the shift in the curves obtained above carbonate sites is in excellent agreement with the MD simulations.

\section{CONCLUSIONS}

In conclusion, we have identified individual cations at the solid-liquid interface by the subtle difference in their hydration structure. The capability of dynamic AFM to provide chemical identification of single atoms at the solid-water interface allows for unraveling so far undiscovered insights into the reactivity of aqueous interfaces. As aqueous interfaces are ubiquitous both in nature and technology, we anticipate that this will have a significant impact on both research and technological development.

\section{ASSOCIATED CONTENT}

\section{S Supporting Information}

The Supporting Information is available free of charge on the ACS Publications website at DOI: 10.1021/acs.langmuir.6b03814

Description of hydration layer assignment, figures showing additional lateral slices as well as the tipsample force gradient and force curves, table containing parameters of the MD simulation (PDF)

\section{AUTHOR INFORMATION}

\section{Corresponding Author}

*E-mail: kuehnle@uni-mainz.de.

ORCID

Adam S. Foster: 0000-0001-5371-5905

Angelika Kühnle: 0000-0003-1214-1006

Notes

The authors declare no competing financial interest.

\section{ACKNOWLEDGMENTS}

H.S. is a recipient of a DFG-funded position through the Excellence Initiative by the Graduate School Materials Science in Mainz (GSC 266). A.K. gratefully acknowledges financial support by the German Research Foundation (DFG) through Grant No. KU1980/7-1. P.S., J.T., and A.S.F. have been supported by the Academy of Finland through its Centres of Excellence Program (Project No. 915804) and acknowledge the use of the computational resources provided by the Aalto Science-IT project. The collaboration between the groups of A.S.F. and A.K. is funded through travel grants from the Academy of Finland (PSINAS, Project No. 11285128) and the Deutscher Akademischer Austausch Dienst (PSINAS, Project No. 57161955).

\section{REFERENCES}

(1) Albrecht, T. R.; Grütter, P.; Horne, D.; Rugar, D. Frequency Modulation Detection Using High-Q Cantilevers for Enhanced Force Microscope Sensitivity. J. Appl. Phys. 1991, 69, 668-673.

(2) Giessibl, F. J. Advances in Atomic Force Microscopy. Rev. Mod. Phys. 2003, 75, 949-983.

(3) Giessibl, F. J. Atomic Resolution of the Silicon $(111)-(7 \times 7)$ Surface by Atomic Force Microscopy. Science 1995, 267, 68-71.

(4) Fukuma, T.; Kobayashi, K.; Matsushige, K.; Yamada, H. True Atomic Resolution in Liquid by Frequency-Modulation Atomic Force Microscopy. Appl. Phys. Lett. 2005, 87, 034101.

(5) Lantz, M. A.; Hug, H. J.; Hoffmann, R.; van Schendel, P. J. A.; Kappenberger, P.; Martin, S.; Baratoff, A.; Güntherodt, H.-J. Quantitative Measurement of Short-Range Chemical Bonding Forces. Science 2001, 291, 2580-2583.

(6) Foster, A. S.; Barth, C.; Shluger, A. L.; Reichling, M. Unambiguous Interpretation of Atomically Resolved Force Microscopy Images of an Insulator. Phys. Rev. Lett. 2001, 86, 2373-2376.

(7) Hoffmann, R.; Lantz, M. A.; Hug, H. J.; van Schendel, P. J. A.; Kappenberger, P.; Martin, S.; Baratoff, A.; Güntherodt, H.-J. Atomic Resolution Imaging and Frequency versus Distance Measurements on 
$\mathrm{NiO}(001)$ Using Low-Temperature Scanning Force Microscopy. Phys. Rev. B 2003, 67, 085402.

(8) Langkat, S. M.; Hölscher, H.; Schwarz, A.; Wiesendanger, R. Determination of Site Specific Interatomic Forces between an Iron Coated Tip and the $\mathrm{NiO}(001)$ Surface by Force Field Spectroscopy. Surf. Sci. 2003, 527, 12-20.

(9) Hoffmann, R.; Weiner, D.; Schirmeisen, A.; Foster, A. S. Sublattice Identification in Noncontact Atomic Force Microscopy of the $\mathrm{NaCl}(001)$ Surface. Phys. Rev. B 2009, 80, 115426.

(10) Rahe, P.; Schütte, J.; Kühnle, A. NC-AFM Contrast Formation on the Calcite $(1 \overline{0} 14)$ Surface. J. Phys.: Condens. Matter 2012, 24, 084006.

(11) Sugimoto, Y.; Pou, P.; Abe, M.; Jelinek, P.; Pérez, R.; Morita, S.; Custance, Ó. Chemical Identification of Individual Surface Atoms by Atomic Force Microscopy. Nature 2007, 446, 64-67.

(12) Watkins, M.; Shluger, A. L. Mechanism of Contrast Formation in Atomic Force Microscopy in Water. Phys. Rev. Lett. 2010, 105, 196101.

(13) Brown, G. E., Jr. How Minerals React with Water. Science 2001, 294, 67-69.

(14) Brown, G. E., Jr.; Calas, G. Mineral-Aqueous Solution Interfaces and Their Impact on the Environment. Geochem. Perspec. 2012, 483742.

(15) Putnis, A. Why Mineral Interfaces Matter. Science 2014, 343, 1441-1442.

(16) Björneholm, O.; Hansen, M. H.; Hodgson, A.; Liu, L.-M.; Limmer, D. T.; Michaelides, A.; Pedevilla, P.; Rossmeisl, J.; Shen, H.; Tocci, G.; Tyrode, E.; Walz, M.-M.; Werner, J.; Bluhm, H. Water at Interfaces. Chem. Rev. 2016, 116, 7698-7726.

(17) Rode, S.; Oyabu, N.; Kobayashi, K.; Yamada, H.; Kühnle, A.

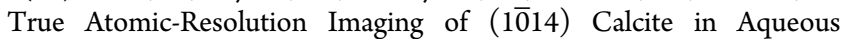
Solution by Frequency Modulation Atomic Force Microscopy. Langmuir 2009, 25, 2850-2853.

(18) Fukuma, T.; Ueda, Y.; Yoshioka, S.; Asakawa, H. Atomic-Scale Distribution of Water Molecules at the Mica-Water Interface Visualized by Three-Dimensional Scanning Force Microscopy. Phys. Rev. Lett. 2010, 104, 016101.

(19) Imada, H.; Kimura, K.; Onishi, H. Water and 2-Propanol Structured on Calcite (104) Probed by Frequency-Modulation Atomic Force Microscopy. Langmuir 2013, 29, 10744-10751.

(20) Fukuma, T.; Reischl, B.; Kobayashi, N.; Spijker, P.; Canova, F. F.; Miyazawa, K.; Foster, A. S. Mechanism of Atomic Force Microscopy Imaging of Three-Dimensional Hydration Structures at a Solid-Liquid Interface. Phys. Rev. B 2015, 92, 155412.

(21) Martin-Jimenez, D.; Chacon, E.; Tarazona, P.; Garcia, R. Atomically Resolved Three-Dimensional Structures of Electrolyte Aqueous Solutions near a Solid Surface. Nat. Commun. 2016, 7, 12164. (22) Hiasa, T.; Kimura, K.; Onishi, H.; Ohta, M.; Watanabe, K.; Kokawa, R.; Oyabu, N.; Kobayashi, K.; Yamada, H. Aqueous Solution Structure over $\alpha-\mathrm{Al}_{2} \mathrm{O}_{3}(01 \overline{1} 2)$ Probed by Frequency-Modulation Atomic Force Microscopy. J. Phys. Chem. C 2010, 114, 21423-21426.

(23) Suzuki, K.; Oyabu, N.; Kobayashi, K.; Matsushige, K.; Yamada, $\mathrm{H}$. Atomic-Resolution Imaging of Graphite-Water Interface by Frequency Modulation Atomic Force Microscopy. Appl. Phys. Express 2011, 4, 125102.

(24) Hiasa, T.; Kimura, K.; Onishi, H. Two-Dimensional Distribution of Liquid Hydrocarbons Facing Alkanethiol Monolayers Visualized by Frequency Modulation Atomic Force Microscopy. Colloids Surf., A 2012, 396, 203-207.

(25) Hiasa, T.; Kimura, K.; Onishi, H. Hydration of Hydrophilic Thiolate Monolayers Visualized by Atomic Force Microscopy. Phys. Chem. Chem. Phys. 2012, 14, 8419.

(26) Nishioka, R.; Hiasa, T.; Kimura, K.; Onishi, H. Specific Hydration on $p$-Nitroaniline Crystal Studied by Atomic Force Microscopy. J. Phys. Chem. C 2013, 117, 2939-2943.

(27) Rode, S.; Stark, R.; Lübbe, J.; Tröger, L.; Schütte, J.; Umeda, K.; Kobayashi, K.; Yamada, H.; Kühnle, A. Modification of a Commercial Atomic Force Microscopy for Low-Noise, High-Resolution Fre-
quency-Modulation Imaging in Liquid Environment. Rev. Sci. Instrum. 2011, 82, 073703.

(28) Adam, H.; Rode, S.; Schreiber, M.; Kobayashi, K.; Yamada, H.; Kühnle, A. Photothermal Excitation Setup for a Modified Commercial Atomic Force Microscope. Rev. Sci. Instrum. 2014, 85, 023703.

(29) Söngen, H.; Nalbach, M.; Adam, H.; Kühnle, A. ThreeDimensional Atomic Force Microscopy Mapping at the Solid-Liquid Interface with Fast and Flexible Data Acquisition. Rev. Sci. Instrum. 2016, 87, 063704.

(30) Lübbe, J.; Temmen, M.; Rahe, P.; Kühnle, A.; Reichling, M. Determining Cantilever Stiffness from Thermal Noise. Beilstein J. Nanotechnol. 2013, 4, 227-233.

(31) Kuhn, S.; Kittelmann, M.; Sugimoto, Y.; Abe, M.; Kühnle, A.; Rahe, P. Identifying the Absolute Orientation of a Low-Symmetry Surface in Real Space. Phys. Rev. B 2014, 90, 195405.

(32) Sader, J. E.; Jarvis, S. P. Accurate Formulas for Interaction Force and Energy in Frequency Modulation Force Spectroscopy. Appl. Phys. Lett. 2004, 84, 1801.

(33) Sader, J. E.; Uchihashi, T.; Higgins, M. J.; Farrell, A.; Nakayama, Y.; Jarvis, S. P. Quantitative Force Measurements Using Frequency Modulation Atomic Force Microscopy - Theoretical Foundations. Nanotechnology 2005, 16, S94-S101.

(34) Hölscher, H. Quantitative Measurement of Tip-Sample Interactions in Amplitude Modulation Atomic Force Microscopy. Appl. Phys. Lett. 2006, 89, 123109.

(35) Plimpton, S. Fast Parallel Algorithms for Short-Range Molecular Dynamics. J. Comput. Phys. 1995, 117, 1-19.

(36) Humphrey, W.; Dalke, A.; Schulten, K. VMD: Visual Molecular Dynamics. J. Mol. Graphics 1996, 14, 33-38.

(37) Michaud-Agrawal, N.; Denning, E. J.; Woolf, T. B.; Beckstein, O. MDAnalysis: A Toolkit for the Analysis of Molecular Dynamics Simulations. J. Comput. Chem. 2011, 32, 2319-2327.

(38) Raiteri, P.; Gale, J. D.; Quigley, D.; Rodger, P. M. Derivation of an Accurate Force-Field for Simulating the Growth of Calcium Carbonate from Aqueous Solution: A New Model for the CalciteWater Interface. J. Phys. Chem. C 2010, 114, 5997-6010.

(39) Tomono, H.; Nada, H.; Zhu, F.; Sakamoto, T.; Nishimura, T.; Kato, T. Effects of Magnesium Ions and Water Molecules on the Structure of Amorphous Calcium Carbonate: A Molecular Dynamics Study. J. Phys. Chem. B 2013, 117, 14849-14856.

(40) Raiteri, P.; Demichelis, R.; Gale, J. D. Thermodynamically Consistent Force Field for Molecular Dynamics Simulations of Alkaline-Earth Carbonates and Their Aqueous Speciation. J. Phys. Chem. C 2015, 119, 24447.

(41) Wu, Y.; Tepper, H. L.; Voth, G. A. Flexible Simple Point-Charge Water Model with Improved Liquid-State Properties. J. Chem. Phys. 2006, 124, 024503.

(42) Kerisit, S.; Parker, S. C. Free Energy of Adsorption of Water and Calcium on the $\{10 \overline{1} 4\}$ Calcite Surface. Chem. Commun. 2004, 52-53.

(43) Perry, T. D., IV; Cygan, R. T.; Mitchell, R. Molecular Models of a Hydrated Calcite Mineral Surface. Geochim. Cosmochim. Acta 2007, $71,5876-5887$

(44) de Leeuw, N. H.; Parker, S. C. Surface-water Interactions in the Dolomite Problem. Phys. Chem. Chem. Phys. 2001, 3, 3217-3221.

(45) Watkins, M.; Reischl, B. A Simple Approximation for Forces Exerted on an AFM Tip in Liquid. J. Chem. Phys. 2013, 138, 154703.

(46) Amano, K.-i.; Liang, Y.; Miyazawa, K.; Kobayashi, K.; Hashimoto, K.; Fukami, K.; Nishi, N.; Sakka, T.; Onishi, H.; Fukuma, T. Number Density Distribution of Solvent Molecules on a Substrate: A Transform Theory for Atomic Force Microscopy. Phys. Chem. Chem. Phys. 2016, 18, 15534-15544.

(47) Miyazawa, K.; Kobayashi, N.; Watkins, M.; Shluger, A. L.; Amano, K.-i.; Fukuma, T. A Relationship between Three-Dimensional Surface Hydration Structures and Force Distribution Measured by Atomic Force Microscopy. Nanoscale 2016, 8, 7334-7342. 